\title{
COMPUTATIONAL STUDY OF FAST METHODS FOR THE EIKONAL EQUATION*
}

\author{
PIERRE A. GREMAUD ${ }^{\dagger}$ AND CHRISTOPHER M. KUSTER ${ }^{\dagger}$
}

\begin{abstract}
A computational study of the fast marching and the fast sweeping methods for the eikonal equation is given. It is stressed that both algorithms should be considered as "direct" (as opposed to iterative) methods. On realistic grids, fast sweeping is faster than fast marching for problems with simple geometry. For strongly nonuniform problems and/or complex geometry, the situation may be reversed. Finally, fully second order generalizations of methods of this type for problems with obstacles are proposed and implemented.
\end{abstract}

Key words. Hamilton-Jacobi, eikonal, viscosity solution, fast marching, fast sweeping

AMS subject classifications. 65N06, 65Y20, 49L25, 35F30

DOI. $10.1137 / 040605655$

1. Introduction. Steady state Hamilton-Jacobi equations play a major role in countless applications. While the discretization of those equations is approaching maturity (at least for single equations and convex Hamiltonians), the resolution of the nonlinear discretized systems is an active topic of research.

In this paper, two methods are compared: the fast marching (FM) method [13, $14,17]$ and the fast sweeping (FS) method $[4,8,16,19]$. The two-dimensional eikonal equation is taken as a test problem: given a slowness field $F$ (a positive function), we look for a time of propagation $u$ satisfying

$$
\begin{aligned}
|\nabla u|=F & \text { in } \Omega \subset \mathrm{R}^{2}, \\
u & =0 \quad \text { on } \Gamma \subset \mathrm{R}^{2} .
\end{aligned}
$$

The two-dimensional domain $\Omega$ is the computational domain; $\Gamma$ is a source, i.e., a closed subset of $\bar{\Omega}$ in which $u$ is zero. The slowness field $F$ can be unbounded, corresponding to the presence of obstacles.

We refer the reader to $[8,15]$ for issues related to the application of the above methods to the more general problem

$$
H(x, \nabla u)=F .
$$

While clearly of importance, those considerations are related more to discretization than they are to resolution and are not the focus of this paper. Discretization algorithms are only briefly reviewed in section 2 . In section 3 , three nonlinear solvers are described (Gauss-Jacobi, FM, and FS/nonlinear Gauss-Seidel). Section 4 presents an adaption of the FM and FS methods to problems with obstacles. The relative efficiency of FM and FS is discussed in section 5. Concluding remarks are offered in section 6 .

\footnotetext{
*Received by the editors March 25, 2004; accepted for publication (in revised form) February 4, 2005; published electronically February 3, 2006.

http://www.siam.org/journals/sisc/27-6/60565.html

${ }^{\dagger}$ Department of Mathematics and Center for Research in Scientific Computation, North Carolina State University, Raleigh, NC 27695-8205 (gremaud@unity.ncsu.edu, cmkuster@unity.ncsu.edu). The work of the first author was partially supported by National Science Foundation grants DMS-0204578, DMS-0244488, and DMS-0410561. The work of the second author was partially supported by National Science Foundation grant DMS-0244488.
} 
2. Discretization. The basic construction principles for numerical Hamiltonians are well known [10] and are easily described on a Cartesian grid. There, the basic scheme takes the form

$$
\mathcal{H}\left(D_{+}^{x} U_{i j}, D_{-}^{x} U_{i j} ; D_{+}^{y} U_{i j}, D_{-}^{y} U_{i j}\right)=\mathcal{F}_{i j},
$$

with the obvious notation. In that case, we recall that $\mathcal{H}$ is consistent if

$$
\mathcal{H}(p, p ; q, q)=H(p, q) \quad \forall p, q,
$$

where $H$ is the Hamiltonian of the problem under consideration (here $H(p, q)=$ $\sqrt{p^{2}+q^{2}}$ ). The numerical Hamiltonian is monotone if nonincreasing in its first and third arguments and nondecreasing in the other two: $\mathcal{H}(\downarrow, \uparrow, \downarrow, \uparrow)[6]$. Consistency ensures that the correct problem is approximated while monotonicity guarantees convergence to the correct viscosity solution $[5,12]$.

Many numerical Hamiltonians can be found in the literature; most of them are derived from corresponding numerical fluxes for conservation laws. The main examples are the Godunov flux $\mathcal{H}^{G}$ and the Lax-Friedrichs flux $\mathcal{H}^{L F}$, which here take the form

$$
\begin{aligned}
\mathcal{H}^{G}\left(p_{+}, p_{-} ; q_{+}, q_{-}\right) & =\sqrt{\max \left\{p_{-}^{+}, p_{+}^{-}\right\}^{2}+\max \left\{q_{-}^{+}, q_{+}^{-}\right\}^{2}}, \\
\mathcal{H}^{L F}\left(p_{+}, p_{-} ; q_{+}, q_{-}\right) & =\sqrt{\left(\frac{p_{+}+p_{-}}{2}\right)^{2}+\left(\frac{q_{+}+q_{-}}{2}\right)^{2}} \\
& -\sigma_{x} \frac{p_{+}-p_{-}}{2}-\sigma_{y} \frac{q_{+}-q_{-}}{2}
\end{aligned}
$$

where $\sigma_{x} \geq \max \left|\frac{\partial H}{\partial p}\right|$ and $\sigma_{y} \geq \max \left|\frac{\partial H}{\partial q}\right|$, where $(\cdot)^{+}=\max \{\cdot, 0\}$ and $(\cdot)^{-}=$ $-\min \{\cdot, 0\}$. Upwind discretizations such as the Godunov method are especially simple to implement for (1.1) and for convex Hamiltonians in general. However, $\mathcal{H}^{G}$, for instance, becomes very involved for general Hamiltonians. While centered methods such as Lax-Friedrichs do not suffer from that problem, they require special treatment at the boundary of the computational domain [8]. The discretization used below is essentially $\mathcal{H}^{G}$.

Many applications call for the use of non-Cartesian or unstructured meshes; see $[1,7,9,14,15]$ for a few examples of such methods. Following $[14,15]$, consider a node $X_{0}$ at which the solution $U$ is to be computed and two neighboring nodes $X_{1}$ and $X_{2}$ at which the values of $U\left(U_{\ell}, \ell=1,2\right)$ and its derivatives $\left(\partial_{x} U_{\ell}, \partial_{y} U_{\ell}, \ell=1,2\right)$ are known or have already been computed. We set

$$
N_{\ell}=\frac{X_{0}-X_{\ell}}{\left|X_{0}-X_{\ell}\right|}, \quad \ell=1,2, \quad \text { and } \quad \mathcal{N}=\left[\frac{N_{1}}{N_{2}}\right]
$$

where $\mathcal{N}$ is a $2 \times 2$ nonsingular matrix, assuming $X_{0}, X_{1}$, and $X_{2}$ are not lined up. The directional derivatives in the directions $N_{1}$ and $N_{2}$ are approximated by

$$
\begin{aligned}
& D_{\ell} U=\frac{U_{0}-U_{\ell}}{\left|X_{0}-X_{\ell}\right|}, \quad \ell=1,2 \quad \text { (first order formula), } \\
& D_{\ell} U=2 \frac{U_{0}-U_{\ell}}{\left|X_{0}-X_{\ell}\right|}-N_{\ell} \cdot\left[\partial_{x} U_{\ell}, \partial_{y} U_{\ell}\right], \quad \ell=1,2 \quad \text { (second order formula). }
\end{aligned}
$$


Those approximate directional derivatives are linked to the gradient by

$$
D_{\ell} U=N_{\ell} \cdot \nabla U+\mathcal{O}\left(\left|X_{0}-X_{i}\right|^{\alpha}\right) \quad \text { or } \quad D U=\mathcal{N} \nabla U+\mathcal{O}\left(h^{\alpha}\right),
$$

where $D U=\left[D_{1} U, D_{2} U\right], h=\max \left\{\left|X_{0}-X_{1}\right|,\left|X_{0}-X_{2}\right|\right\}$, and $\alpha=1$ or 2 depending on whether (2.3) or (2.4), respectively, is used. Solving for $\nabla U$ and plugging into (1.1), one finds the (quadratic) equation defining the unknown $U_{0}$,

$$
D U^{t}\left(\mathcal{N N}^{t}\right)^{-1} D U=\left(F\left(X_{0}\right)\right)^{2} .
$$

The above methods are well understood theoretically: convergence to the viscosity solution when the Godunov flux $\mathcal{H}^{G}$ is used was established in [12]; see also [15] for additional results and references.

3. Resolution. The system of coupled quadratic equations corresponding to imposing (2.6) at all nodes has to be solved. If $\theta$ denotes the angles between $N_{1}$ and $N_{2}$, the matrix $\mathcal{N N}^{t}$ is symmetric positive definite provided $0<\theta<\pi$; its condition number is $\frac{1+\cos \theta}{1-\cos \theta}$. Therefore, regardless of the choice of stencil (first or second order), (2.6) has two real solutions. This situation is generic for Hamilton-Jacobi equations.

Let $\left(U_{1}, U_{2}, \ldots, U_{N}\right)$ be the unknowns for an arbitrary ordering of the nodes. The resulting system can be written symbolically as

$$
f(U)=f\left(U_{1}, U_{2}, \ldots, U_{N}\right)=0 .
$$

An iterative scheme, essentially a fixed point method or Gauss-Jacobi method, was proposed in [12]. One step of the algorithm is as follows.

Gauss-JACOBI ALGORITHM.

- Choose a node and regard the neighboring values as fixed.

- Solve (2.6) for the value at the considered node; the largest of the two possible solutions is selected, in agreement with the viscosity criterion.

- Repeat until convergence (fixed point).

The corresponding pseudocode is

$$
\begin{aligned}
& \mathrm{k}=0 \\
& \mathrm{U}^{0}=(0, \ldots, 0) \\
& \text { while } \mathrm{U}^{\mathrm{k}} \neq \mathrm{U}^{\mathrm{k}+1} \text { or } \mathrm{k} \neq 0 \\
& \mathrm{k}=\mathrm{k}+1 \\
& \text { for } \ell=1 \text { to } N \\
& \text { solve } \mathrm{f}\left(\mathrm{U}_{1}^{\mathrm{k}-1}, \ldots, \mathrm{U}_{\ell-1}^{\mathrm{k}-1}, \mathrm{U}_{\ell}^{\mathrm{k}}, \mathrm{U}_{\ell+1}^{\mathrm{k}-1}, \ldots, \mathrm{U}_{\mathrm{N}}^{\mathrm{k}-1}\right)=0 \text { for } \mathrm{U}_{\ell}^{\mathrm{k}} \\
& \text { end }
\end{aligned}
$$

This approach is obviously slow, as each node has to be revisited several times before the numerical solution settles down. Further, no advantage is taken of the propagation character of the problem.

With marching methods, the nodes are considered in an order consistent with the way the wave fronts propagate, i.e., with the Huygens principle $[13,14,17]$. This leads to (quasi) single pass algorithms. The fast marching algorithm, FM, is based on the monotonicity of the solution along the characteristics. The nodes are divided into three sets: the already-computed nodes, the not-yet-considered nodes, and the nodes "on the propagation front." 
FAST Marching ALGORIThm.

- The node with lowest value in the "front" set is removed from it and added to the "computed" set; its not-yet-computed neighbors are added to the "front."

- The values of all the nodes in the "front" are recomputed using (2.6).

- Repeat until all the nodes are "computed."

The list operations are handled by the heap sort algorithm $[3,18]$, which is the key to the speed of FM. This type of sort has the advantage that every tree operation (insertion, removal, update) is of order $\log _{2} n$, where $n$ is the number of elements in the tree, i.e., the number of "front" nodes at that step. Note that $n$ is of order $\sqrt{N}$ at every step, where $N$ is the total number of nodes in the mesh. By placing the "front" nodes into this structure, the computational complexity is kept at the order of $N \log _{2} N$. The pseudocode for FM is

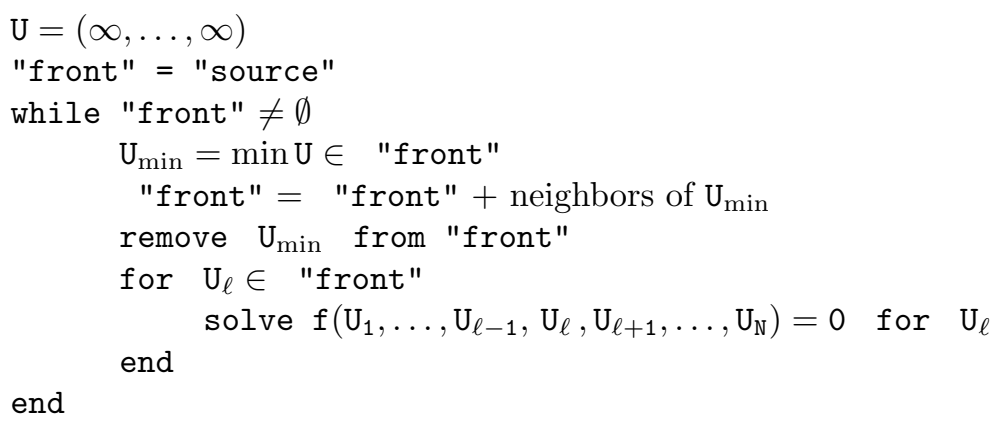

A third type of method has recently been proposed, the fast sweeping (FS) method; see among others $[4,8,16,19]$. FS aims at improving on the first GaussJacobi method by using a Gauss-Seidel-type update process. This change alone would not improve on the performance of Gauss-Jacobi very significantly because of the upwind condition (see (4.1) below): an ordering process is needed. Instead of proceeding in a way consistent with the underlying wave propagation, FS considers sweeps in predetermined directions. The directions of sweep can correspond, for instance, to the direction of the coordinate axes. For two-dimensional problems, four sweeping directions can be considered. More precisely, in an $M \times M$ matrix, we would have $i=1: M, j=1: M$ (upper left to lower right, ULLR); $j=1: M, i=M: 1$ (lower left to upper right, LLUR); $i=M: 1, j=M: 1$ (lower right to upper left, LRUL); $j=M: 1, i=1: M$ (upper right to lower left, URLL).

Fast SweEPING ALgORITHM.

- Choose a direction of sweep and a corresponding ordering.

- Loop through of all the nodes in the chosen order and successively solve (2.6) for each of them.

- Repeat for the other directions of sweep.

- Repeat until convergence.

The corresponding pseudocode is

$$
\begin{aligned}
& \mathrm{k}=0 \\
& \mathrm{U}^{0}=(\infty, \ldots, \infty) \\
& \text { order }=[\mathrm{ULLR}, \mathrm{LLUR}, \text { LRUL.URLL }] \\
& \begin{aligned}
\text { while } \mathrm{U}^{\mathrm{k}} \neq \mathrm{U}^{\mathrm{k}+1} \text { or } \mathrm{k}=0 \\
\mathrm{k}=\mathrm{k}+1 \\
\quad \mathrm{i}=\bmod (\mathrm{k}-1,4)+1 \\
\quad \text { ordering }=\operatorname{order}[\mathrm{i}]
\end{aligned}
\end{aligned}
$$




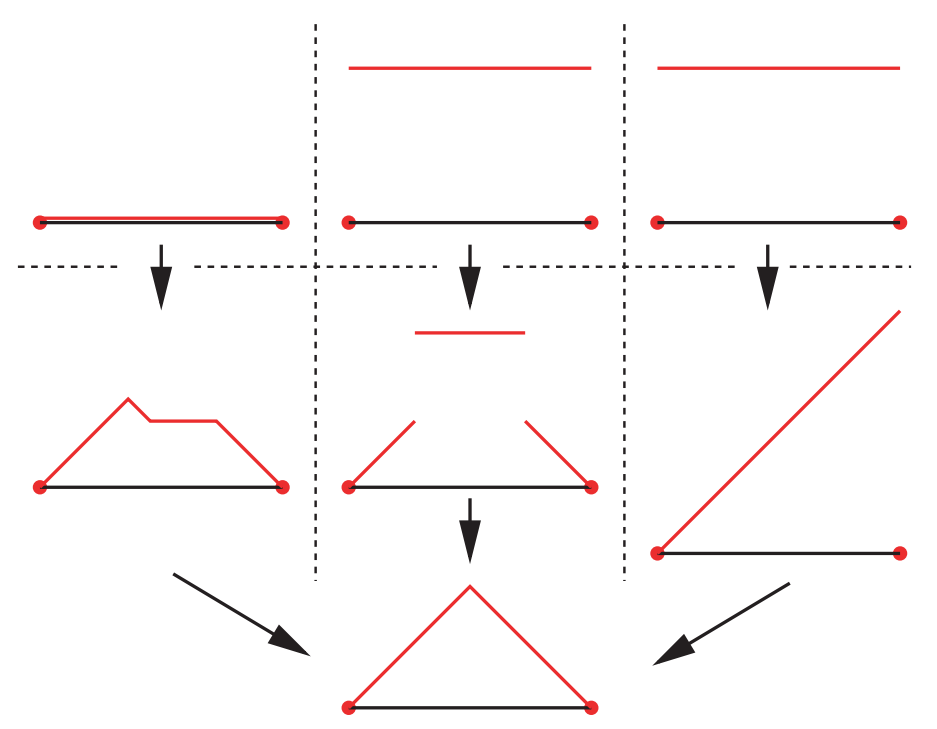

FIG. 1. Schematic representation of the update process for Gauss-Jacobi (left), FM (center), and FS (right) for a one-dimensional eikonal equation with homogenous boundary conditions.

$$
\begin{aligned}
& \text { for } \ell=1 \text { to } N \\
& \text { solve } f\left(U_{1}^{\mathrm{k}}, \ldots, \mathrm{U}_{\ell-1}^{\mathrm{k}}, \mathrm{U}_{\ell}^{\mathrm{k}}, \mathrm{U}_{\ell+1}^{\mathrm{k}-1}, \ldots, \mathrm{U}_{\mathrm{N}}^{\mathrm{k}-1}\right)=0 \text { for } \mathrm{U}_{\ell}^{\mathrm{k}} \\
& \text { end }
\end{aligned}
$$

end

The update process for the above three methods is schematically represented in Figure 1. Also, as a result of the absence of a true ordering process (only the way arrays are accessed changes), the complexity of the above algorithm can be shown to be of order $N$ [19], as opposed to $N \log _{2} N$ for FM. However, whether this translates into a computational advantage depends on the geometry of the problem; see section 5 .

Unlike what is proposed in $[16,19]$, we consider FS as a direct method for two reasons. First, as shown in section 4 , the algorithm has to be run to its completion. Second, the following result gives a natural stopping criterion.

LEMma 3.1. Let $U^{k}, k=0, \ldots, \infty$, be the iterates generated by the FS method applied to (1.1), (1.2). Then there exists $\bar{k}$ such that $U^{k}=U^{\bar{k}}$ for all $k \geq \bar{k}$.

Proof. Consider an arbitrary node $i$ at which the solution has been computed. There exists a path made of successive upwind neighbors from $i$ to $\Gamma$. By construction, each successive sweep determines the final value of at least one node on that path, starting on $\Gamma$ and moving successively to $i$. This clearly leads to a finite algorithm. The numerical solution at node $i$ depends only on the values at its neighbors. If those values do not change at step $\bar{k}+1$, then $U_{i}^{k}=U_{i}^{\bar{k}}$ for $k \geq \bar{k}$.

Essentially, the three methods differ in the order in which the nodes are computed. Gauss-Jacobi takes no particular order, FM uses an ordering based on partial numerical solutions, while FS uses a mesh-based ordering. As described above, FS makes full use of the Cartesian structure of the mesh.

4. Problems with obstacles and implementation. An obstacle is a domain in which waves propagate at infinitely slow speed: $F(x, y)=\infty$ if and only if $(x, y)$ belongs to the obstacle. Problems with obstacles require additional care if both accuracy and efficiency, i.e., marching character, of the methods are to be preserved. 
For future reference, we now introduce three specific test problems used in this paper. In all three cases, we consider $(1.1),(1.2)$ with $\Omega=(0,6)^{2}, \Gamma=(0,0)$.

Example 1. $F(x, y)=C$ for all $(x, y) \in \Omega$, no obstacle. ${ }^{1}$

Example 2.

$$
F(x, y)= \begin{cases}\infty & \text { if }(x-3)^{2}+(y-3)^{2}<=1 \\ C & \text { otherwise }\end{cases}
$$

circular obstacle.

Example 3.

$$
F(x, y)= \begin{cases}\infty & \text { if }(x, y) \in \Xi \\ C & \text { otherwise }\end{cases}
$$

where $\Xi$ is the "rings" of Figure 7 .

If no local adaption is made when treating problems with obstacles such as Examples 2 and 3, accuracy is lost. Taking, for instance, Example 2, a straightforward discretization would consist of applying any of the above three methods outside the circular obstacle using a simple Cartesian mesh. The left half of Table 1 illustrates the loss accuracy incurred by the first and second order FM methods, respectively, i.e., using (2.3) and (2.4).

TABLE 1

Convergence study for formally first and second order methods in the presence of a circular obstacle (Example 2); $M$ measures the number of nodes on one edge of $\Omega$, i.e., total number of

\begin{tabular}{|c|c|c|c|c|c|c|c|c|c|c|c|c|}
\hline \multicolumn{13}{|c|}{ First order methods } \\
\hline & \multicolumn{6}{|c|}{ Standard method } & \multicolumn{6}{|c|}{ Modified method } \\
\hline$M$ & $L^{1}$ & Rate & $L^{2}$ & Rate & $L^{\infty}$ & Rate & $L^{1}$ & Rate & $L^{2}$ & Rate & $L^{\infty}$ & Rate \\
\hline 25 & $2.91(0)$ & & $9.00(-1)$ & & $6.11(-1)$ & & $1.13(0)$ & & $3.04(-1)$ & & $1.44(-1)$ & \\
\hline 50 & $1.71(0)$ & .77 & $5.24(-1)$ & .78 & $3.52(-1)$ & .79 & $7.58(-1)$ & .58 & $2.00(-1)$ & .60 & $9.18(-2)$ & .65 \\
\hline 100 & $1.07(0)$ & .68 & $3.27(-1)$ & .68 & $2.23(-1)$ & .66 & $4.41(-1)$ & .78 & $1.13(-1)$ & .82 & $4.92(-2)$ & .88 \\
\hline 200 & $6.42(-1)$ & .74 & $2.00(-1)$ & .71 & $1.46(-1)$ & .61 & $2.39(-1)$ & .88 & $6.10(-2)$ & .89 & $2.65(-2)$ & .91 \\
\hline 400 & $3.76(-1)$ & .77 & $1.20(-1)$ & .74 & $9.36(-2)$ & .64 & $1.27(-1)$ & .92 & $3.24(-2)$ & .91 & $2.02(-2)$ & .39 \\
\hline 800 & $2.21(-1)$ & .77 & $7.24(-2)$ & .73 & $6.07(-2)$ & .62 & $6.38(-2)$ & .99 & $1.64(-2)$ & .99 & $1.54(-2)$ & .39 \\
\hline 1600 & $1.31(-1)$ & .76 & $4.40(-2)$ & .72 & $3.82(-2)$ & .67 & $3.07(-2)$ & 1.1 & $7.91(-3)$ & 1.0 & $1.14(-2)$ & 43 \\
\hline 3200 & $7.80(-2)$ & .75 & $2.68(-2)$ & .72 & $2.44(-2)$ & .65 & $1.49(-2)$ & 1.0 & $3.88(-3)$ & 1.0 & $6.77(-3)$ & .75 \\
\hline \multicolumn{13}{|c|}{ Second order methods } \\
\hline & \multicolumn{6}{|c|}{ Standard method } & \multicolumn{6}{|c|}{ Modified method } \\
\hline$M$ & $L^{1}$ & Rate & $L^{2}$ & Rate & $L^{\infty}$ & Rate & $L^{1}$ & Rate & $L^{2}$ & Rate & $L^{\infty}$ & Rate \\
\hline 25 & $2.52(0)$ & & $1.07(0)$ & & $8.99(-1)$ & & $1.17(0)$ & & $2.84(-1)$ & & $1.16(-1)$ & \\
\hline 50 & $1.45(0)$ & .80 & $5.94(-1)$ & .85 & $7.39(-1)$ & .28 & $2.83(-1)$ & 2.0 & $9.59(-2)$ & 1.6 & $4.54(-2)$ & 1.4 \\
\hline 100 & $6.36(-1)$ & 1.2 & $2.45(-1)$ & 1.3 & $3.10(-1)$ & 1.3 & $1.23(-1)$ & 1.2 & $4.26(-2)$ & 1.2 & $1.97(-2)$ & 1.2 \\
\hline 200 & $3.02(-1)$ & 1.1 & $1.20(-1)$ & 1.0 & $1.73(-1)$ & .84 & $3.29(-2)$ & 1.9 & $1.14(-2)$ & 1.9 & $1.22(-2)$ & .70 \\
\hline 400 & $1.52(-1)$ & .99 & $6.16(-2)$ & .97 & $8.49(-2)$ & 1.0 & $1.52(-2)$ & 1.1 & $5.32(-3)$ & 1.1 & $5.93(-3)$ & 1.0 \\
\hline 800 & $6.91(-2)$ & 1.1 & $2.91(-2)$ & 1.1 & $4.41(-2)$ & .95 & $2.06(-3)$ & 2.9 & $8.22(-4)$ & 2.7 & $3.11(-3)$ & .94 \\
\hline 1600 & $3.42(-2)$ & 1.0 & $1.45(-2)$ & 1.0 & $2.31(-2)$ & .93 & $4.82(-4)$ & 2.1 & $2.47(-4)$ & 1.7 & $1.68(-3)$ & .89 \\
\hline 3200 & $1.48(-2)$ & 1.2 & $6.36(-3)$ & 1.2 & $1.12(-2)$ & 1.0 & $1.15(-4)$ & 2.1 & $7.64(-5)$ & 1.7 & $8.83(-4)$ & .93 \\
\hline
\end{tabular}
nodes $N=\mathcal{O}\left(M^{2}\right)$.

As can be seen from those results, the standard first order method converges with an average order of only about .75 (in the $L^{1}$ norm) while the standard formally second order method loses a full order (the average rate in the $L^{1}$ norm is about 1.0).

\footnotetext{
${ }^{1}$ In all the calculations that follow, $C$ was chosen as $\tan 40^{\circ}$; see [2] for a related application to granular piles. This choice does not affect the numerical results.
} 


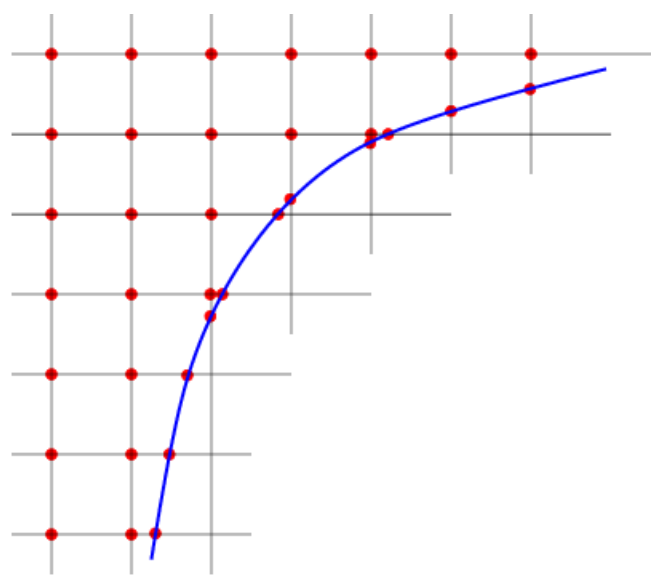

FIG. 2. Mesh structure around the obstacle.

The results in the $L^{2}$ norm are comparable, while the $L^{\infty}$ rates are significantly lower. This is not surprising since the underlying solution presents a "shock" at the back of the obstacle (discontinuity of the first derivatives).

In [2], we started the study of a generalization of the FM method from [14, 15] to problems with obstacles. The nodes are defined on a uniform Cartesian mesh away from the obstacle. Near the obstacle, additional nodes corresponding to the intersection of the obstacle's boundary with the mesh lines are added; see Figure 2. The detailed solving process is as follows for the modified FM algorithm.

Let $X_{0}$ be a node to be updated. Let $X_{1}$ and $X_{2}$ be a pair of primary neighbors of $X_{0}$. Following [15], an upwinding criterion is considered: the characteristic direction should point into the simplex defined by $X_{0}, X_{1}$, and $X_{2}$. This is equivalent to requiring the approximate gradient defined by (2.5) to have positive components when expressed in terms of the unit vectors $N_{1}=\frac{X_{0}-X_{1}}{\left|X_{0}-X_{1}\right|}$ and $N_{2}=\frac{X_{0}-X_{2}}{\left|X_{0}-X_{2}\right|}$. In other words,

$$
\left(\mathcal{N N}^{t}\right)^{-1} D U>0
$$

The value $U_{0}$ at $X_{0}$ is then determined as follows:

$$
U_{0}=\min \left\{\begin{array}{l}
\text { solution of }(2.6) \text { with second order stencil (2.4) if }(4.1) \text { is true, } \\
\text { solution of }(2.6) \text { with first order stencil (2.3) if }(4.1) \text { is true, } \\
\min _{i=1,2}\left\{U_{i}+\left|X_{0}-X_{i}\right| F\left(X_{0}\right)\right\}
\end{array}\right.
$$

Regardless of the choice of stencil (first or second order), (2.6) has two real solutions, from which we always consider the larger one only, to be consistent with causality. In the first two cases, the values of the partial derivatives of $U$ at $X_{0}$ are updated using $\nabla U=\mathcal{N}^{-1} D U$. In the third case, we set $\nabla U=N_{i} F\left(X_{0}\right)$, where $i$ is the index corresponding to the case of lowest value for $U_{0}$. This process is repeated for all pairs of primary neighbors $X_{1}$ and $X_{2}$, and the lowest resulting value of $U_{0}$ is kept.

As checking (4.1) accounts for a sizable part of the runtime, the following result is useful in practice.

Lemma 4.1. Let $\theta, 0<\theta<\pi$, be the angle between $N_{1}$ and $N_{2}$. The upwind condition (4.1) is equivalent to

$$
\left|D_{1} U-D_{2} U\right|<(1-\cos \theta) F\left(X_{0}\right) .
$$


Proof. The result is easy to verify geometrically. Setting $d_{i}=D_{i} U, i=1,2$, the scheme (2.6) takes the form

$$
d_{1}^{2}-2 \cos \theta d_{1} d_{2}+d_{2}^{2}=\sin ^{2} \theta F\left(X_{0}\right)^{2} .
$$

This defines an ellipse in the $d_{1} d_{2}$-plane with major (respectively, minor) semiaxis of length $\frac{\sin \theta}{\sqrt{1-\cos \theta}} F\left(X_{0}\right)$ (respectively, $\frac{\sin \theta}{\sqrt{1+\cos \theta}} F\left(X_{0}\right)$ ) in the direction $[1,1]$ (respectively, $[1,-1])$. The upwind condition $(4.1)$ reads as

$$
d_{1}-\cos \theta d_{2}>0 \text { and } d_{2}-\cos \theta d_{1}>0 .
$$

As Figure 3 and a little algebra show, the portion of the ellipse that satisfies the above conditions can also be characterized by $\left|d_{1}-d_{2}\right|<(1-\cos \theta) F\left(X_{0}\right)$. Note that this last condition also admits a set of solutions opposite the desired ones on the ellipse depicted in Figure 3. Those solutions are, however, never encountered in practice since only the largest of the two roots of the quadratic equation is retained.

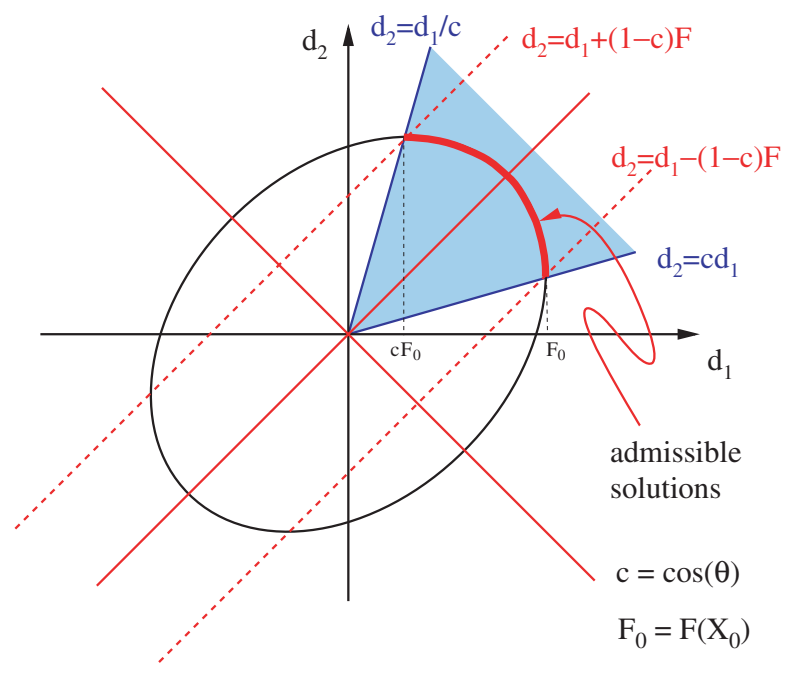

FIG. 3. Upwind condition; see Lemma 4.1 and its proof.

For general meshes, the above condition requires the calculation of $U_{0}$ and has to be checked a posteriori. For a uniform Cartesian mesh of size $\Delta x,(4.3)$ reads as

$$
\left|U_{1}-U_{2}\right|<\Delta x F\left(X_{0}\right)
$$

and can be checked a priori, i.e., without having to solve the quadratic equation (2.6). This explains the large discrepancies in runtimes in favor of the low accuracy method on uniform Cartesian grids reported in Table 2 (see Figure 5 for a comparison of runtime versus accuracy).

As observed in [11], in the presence of a point source, a singularity of the travel time field is clearly located at the source itself. Unless special care is taken near that source, a loss of accuracy ensues. The problem can be fixed by defining a mesh- 
TABLE 2

Runtimes illustrating the overhead due to higher accuracy for the FM method applied to Example 2 ; see text for full details.

\begin{tabular}{|l|c|c|c|}
\hline$M$ & Time 1 & Time 2 & Time 3 \\
\hline 100 & 0.02 & 0.57 & 0.60 \\
\hline 200 & 0.10 & 2.20 & 2.38 \\
\hline 400 & 0.43 & 8.93 & 9.45 \\
\hline 800 & 2.02 & 34.5 & 38.1 \\
\hline 1600 & 10.1 & 143 & 154 \\
\hline 3200 & 53.0 & 610 & 654 \\
\hline
\end{tabular}
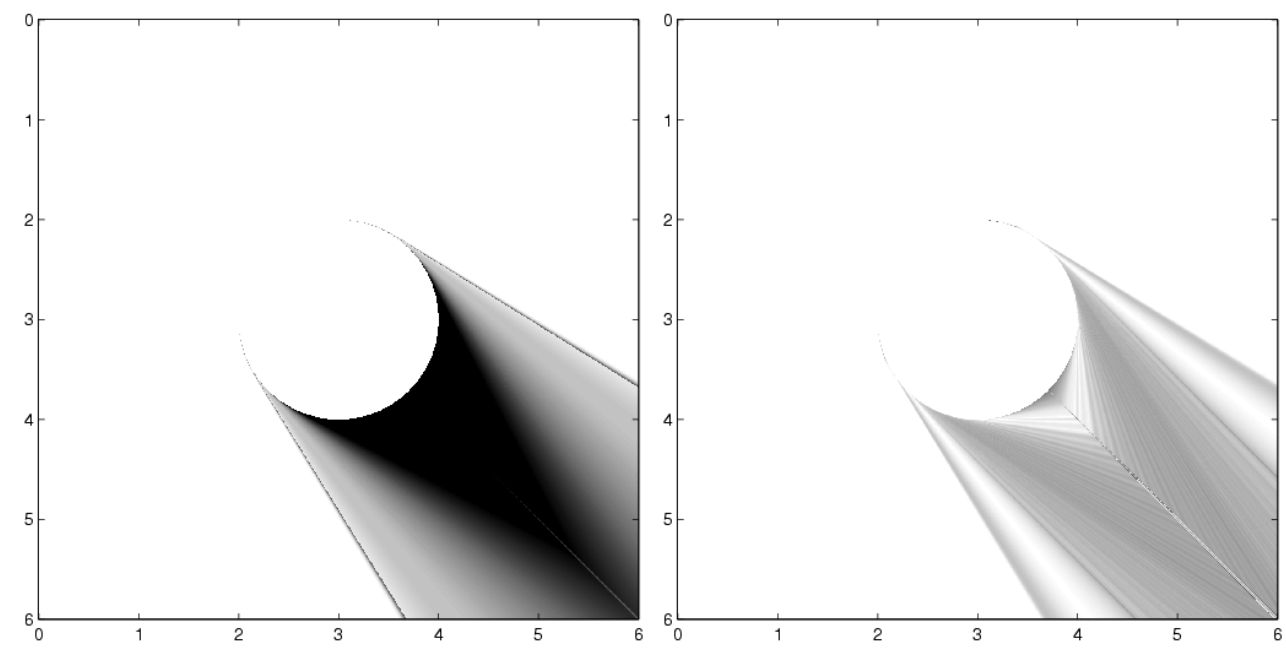

Fig. 4. Error propagation downstream of the obstacle for the standard (left) and modified (right) second order FM method (Example 2, $M=1600, N=\mathcal{O}\left(M^{2}\right)$ ). The source is located at $(0,0)$.

independent domain around the source and initializing the values at the nodes there to the corresponding values of the exact solution. This fix was applied to all methods under study here. As illustrated in Table 1, the modified scheme is found to preserve first and second order accuracy in the $L^{1}$ norm even in the presence of obstacles. For the second order version, the $L^{2}$ rates of convergence are slightly below 2 , while the method appears to converge with order 1 in the maximum norm. In all cases and all norms, the modified method exhibits much better accuracy than the standard method. Error propagation downstream from the obstacle is illustrated in Figure 4 for both standard and modified methods.

It should be noted that second order convergence for the finer meshes was obtained only after rewriting the quadratic formula for solving (2.6) in a form that limits cancellation effects. (Standard IEC559 double precision was used throughout.)

Higher accuracy does not come for free. In Table 2, the overhead corresponding to maintaining optimal accuracy is described in terms of computational time and number of operations.

Table 2 compares three different algorithms. Time 1 corresponds to the computational time and number of operations for the standard method where full advantage has been taken of the uniform Cartesian mesh in the implementation: simplification 


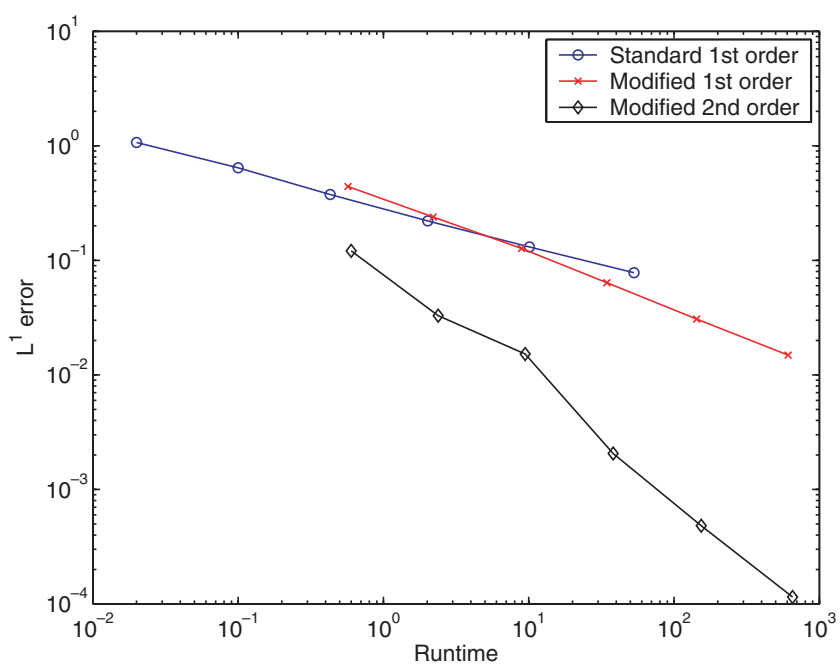

FIG. 5. $L^{1}$ error versus runtime for the standard "first" order method, the modifed first order method, and the modified second order method applied to Example 2.

of the quadratic equation (2.6) and of the upwinding condition (see remarks above). The results for the fully first and second order methods correspond, respectively, to Time 2 and Time 3 . The fully second order method is found to be only marginally slower than the fully first order one. It is clear from Table 2 that a significant price has to be paid for the increase in accuracy. However, Figure 5 shows that the advantage in speed for the standard first order is offset by its relative lack of accuracy and slow convergence as the mesh is refined.

FS as described in [16, 19], for instance, is proposed as an iterative method, i.e., the algorithm is stopped before completion according to a stopping criterion of the type $\left\|u^{k+1}-u^{k}\right\|_{L^{1}}<\delta=10^{-10}$. Figure 6 illustrates the convergence history of the numerical solution toward the exact solution of the discretized problem for Example 3. The discrepancy falls down to zero if one additional step is taken. It is observed that stopping on small decreases, as suggested by the previous criterion, is not advisable. Therefore, running FS until completion appears to be the best strategy, and the algorithm has to be considered as a "direct" method.

For both FM and FS, obstacles can be implemented as domains with truly infinite speed (in which case, no calculations are made at nodes inside the obstacle), or a sufficiently large but finite slowness can be assigned to nodes inside the obstacle and the solution can be computed there. Our implementation uses the former approach. As can be expected and is illustrated in Figure 6, those choices do not affect the solutions of the discretized problems.

5. Algorithms comparison. The three examples from section 4 were implemented. As the implementation on FS on a non-Cartesian grid was not attempted, first order FM and FS were compared on Cartesian grids. Therefore in Table 3, both methods share the same (in)accuracy. Note that each algorithm was run until "convergence." For FM, this means until exhaustion of the nodes in the "front," while for FS it means until a fixed point has been reached (no change after a sweep; see Lemma 3.1). As can be seen from that table, FS is faster for the first (trivial) example; 


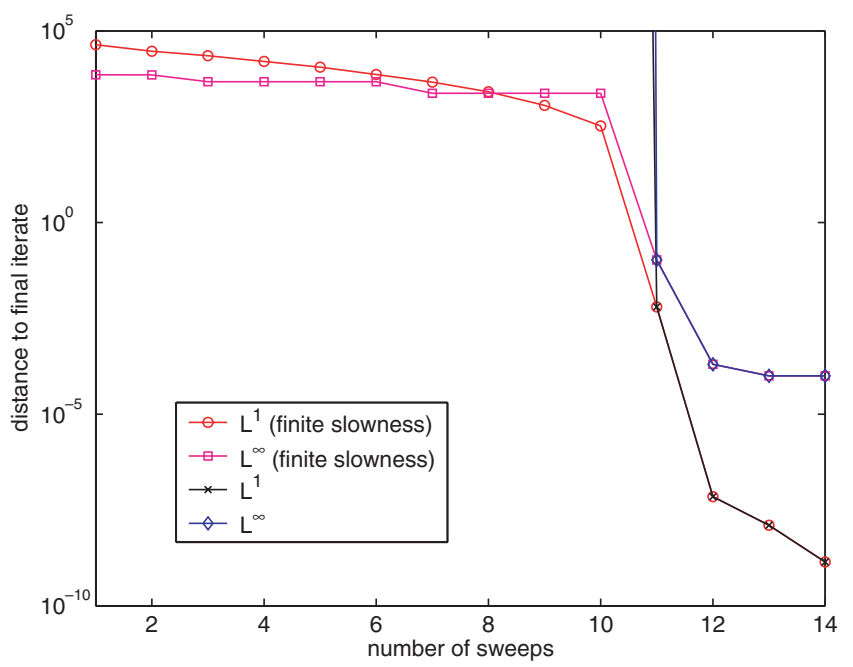

FIG. 6. $L^{1}$ and $L^{\infty}$ norms of the difference between the exact solution of the discretized problem with the FS method and iterates after each sweep; rings (Example 3); $M=1600$. The curves with "finite slowness" are obtained by treating the obstacle as a domain with large slowness; the other curves correspond to a genuine obstacle (slowness $=\infty$ ).

both methods perform in a way very similar to Example 2, with a slight advantage for FS, while on Example $3 \mathrm{FM}$ is faster for all but the finest mesh. ${ }^{2}$

The results from Table 3 also show that, for moderate mesh sizes, the more complicated the domain is, the better FM performs with respect to FS. Indeed, while FM continually advances the wavefront, FS has to do another set of sweeps every time the direction of propagation changes. In the case of Example 3, for instance, that direction changes several times; see Figure 7. In Table 4, the runtimes for a modified Example 3 are reported: the number of partial rings was increased from zero to a total of five as displayed in Figure 7. It is observed that the runtime is roughly constant for FM as the complexity of the domain (number of rings) increases. For FS, however, the runtime increases, although in a nonuniform way. This nonuniformity results from both the geometry of the domain (odd/even number of rings) and our specific implementation.

As argued in [19], FS has an asymptotic computational complexity of $\mathcal{O}(N)$ while FM's is $\mathcal{O}\left(N \log _{2} N\right)$. Eventually, FS overcomes FM, even for nontrivial examples such as Example 3, as the mesh gets fine enough. The constants of proportionality involved in those asymptotic relations are not easy to analyze. For specific examples, those constants can be estimated numerically. Assuming runtimes for FS and FM to be, respectively, equal to $C_{F S} m N$ and $C_{F M} N \log _{2} N$, where $m$ is the number of sweeps required by FS, one can use results similar to those from Table 3 to roughly determine the two constants $C_{F S}$ and $C_{F M}$. An empirical condition under which FS is faster than FM can then be derived. Using best fits to the above examples and cases, one finds here

$$
m \lesssim .6 \log _{2} N .
$$

\footnotetext{
${ }^{2}$ In an initial version of this paper (technical report), the reported runtimes had been obtained by codes that were not fully optimized for use on uniform Cartesian meshes. One of those optimization steps removed unnecessary checks from FS; those redundant operations were, by construction, not present in the FM algorithm. Therefore, the two codes were not equally affected. To the authors' knowledge, the current codes are fully optimized for uniform Cartesian grids.
} 
TABLE 3

Runtime for first order FM and FS on a uniform Cartesian mesh for Examples 1, 2, and 3 $\left(N=\mathcal{O}\left(M^{2}\right)\right)$.

\begin{tabular}{|l|c|c|c|}
\hline$M$ & FM (time) & FS (time) & \# sweeps \\
\hline \multicolumn{4}{|c|}{ Example 1: no obstacle } \\
\hline 100 & 0.01 & 0.01 & 2 \\
\hline 200 & 0.09 & 0.03 & 2 \\
\hline 400 & 0.43 & 0.13 & 2 \\
\hline 800 & 2.03 & 0.54 & 2 \\
\hline 1600 & 10.1 & 2.16 & 2 \\
\hline 3200 & 53.6 & 8.59 & 2 \\
\hline \multicolumn{4}{|c|}{ Example 2: circular obstacle } \\
\hline 100 & 0.02 & 0.01 & 5 \\
\hline 200 & 0.10 & 0.09 & 6 \\
\hline 400 & 0.43 & 0.37 & 6 \\
\hline 800 & 2.02 & 1.45 & 6 \\
\hline 1600 & 10.1 & 5.91 & 6 \\
\hline 3200 & 53.0 & 23.5 & 6 \\
\hline \multicolumn{4}{|c|}{ Example 3: "rings" } \\
\hline 100 & 0.02 & 0.05 & 16 \\
\hline 200 & 0.10 & 0.22 & 16 \\
\hline 400 & 0.48 & 0.91 & 16 \\
\hline 800 & 2.28 & 3.44 & 16 \\
\hline 1600 & 11.4 & 14.3 & 16 \\
\hline 3200 & 57.5 & 56.8 & 16 \\
\hline
\end{tabular}

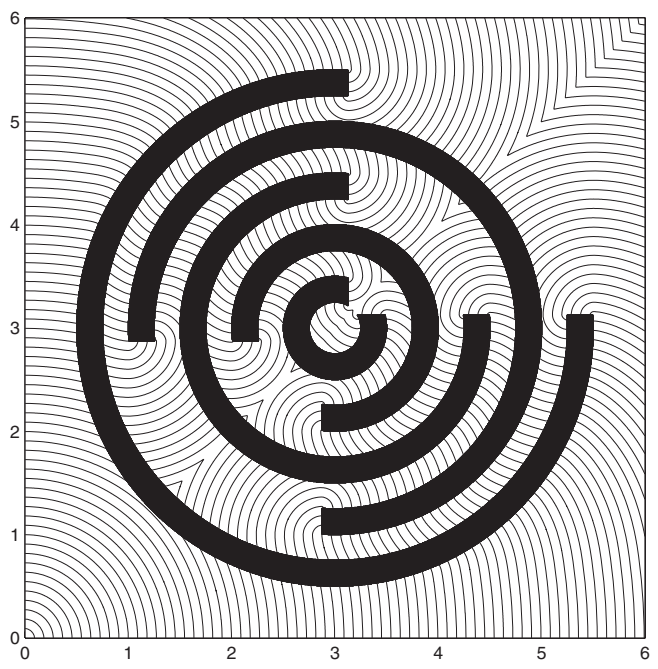

FIG. 7. Propagation around an obstacle: Example 3, FM with $M=800$. The source is located at $(0,0)$.

In other words, for a "reasonable" $800 \times 800$ mesh $\left(N=800^{2}\right)$, FS will be faster than FM as long as the number of sweeps necessary for convergence does not exceed about 11. This is in good agreement with Table 4.

6. Conclusion. We have brought to the fore three main points. First, ordered methods for the eikonal equation can be modified to retain optimal convergence rates (first or second order), even on low regularity problems, while retaining their efficiency. This can be done in a natural way for FM, but it is more cumbersome for FS, which is 
TABLE 4

Runtime and operation count with differing numbers of concentric partial rings $(M=800)$.

\begin{tabular}{|c|c|c|c|}
\hline Rings & FM (time) & FS (time) & \# sweeps \\
\hline 0 & 2.03 & 0.54 & 2 \\
\hline 1 & 2.05 & 2.15 & 8 \\
\hline 2 & 2.12 & 2.09 & 8 \\
\hline 3 & 2.12 & 3.02 & 12 \\
\hline 4 & 2.24 & 2.89 & 12 \\
\hline 5 & 2.28 & 3.44 & 16 \\
\hline
\end{tabular}

much less flexible. Second, the examples considered show that both methods should be thought of as direct and not iterative algorithms. This is obvious for FM. For FS, a natural stopping criterion is proposed and justified. Finally, FS and FM have asymptotic orders of complexity of, respectively, $\mathcal{O}(m N)$ and $\mathcal{O}\left(N \log _{2} N\right)$, where $m$ is the number of sweeps and $N$ is the total number of nodes. On a realistic grid, FS will be faster than FM for problems with simple geometry. For strongly nonuniform problems and/or complex geometry, the situation may be reversed.

Both methods can be relatively easily extended to more general convex Hamiltonians $[15,16]$. Behaviors similar to those reported here are expected. The case of nonconvex Hamiltonians is more complex, even with respect to the construction of appropriate numerical fluxes. Very few schemes have been proposed (see, for instance, [8], where a Lax-Friedrichs sweeping method is introduced); more needs to be done to develop fast and accurate methods for those problems.

Acknowledgments. The authors are grateful to Hongkai Zhao for his helpful comments and to the referees for remarks that led to a better presentation of the results.

\section{REFERENCES}

[1] R. Abgrall, Numerical discretization of the first order Hamilton-Jacobi equations on triangular meshes, Comm. Pure Appl. Math., 49 (1996), pp. 1339-1377.

[2] S.A. Ahmed, R. Buckingham, P.A. Gremaud, C.D. Hauck, C.M. Kuster, M. Prodanovic, T.A. Royal, AND V. Silantyev, Volume determination for bulk materials in bunkers, Internat. J. Numer. Methods Engrg., 61 (2004), pp. 2239-2249.

[3] J. Bentley, Thanks, heaps, Comm. ACM, 28 (1985), pp. 245-250.

[4] M. BouÉ AND P. DupuIS, Markov chain approximations for deterministic control problems with affine dynamics and quadratic cost in the control, SIAM J. Numer. Anal., 36 (1999), pp. 667-695.

[5] M. Crandall, L. Evans, and P.-L. Lions, Some properties of viscosity solutions of HamiltonJacobi equations, Trans. Amer. Math. Soc., 282 (1984), pp, 487-502.

[6] M. Crandall and P.-L. Lions, Two approximations of solutions of Hamilton-Jacobi equations, Math. Comp., 43 (1984), pp. 1-19.

[7] C. Hu AND C.-W. SHU, A discontinuous Galerkin method for Hamilton-Jacobi equations, SIAM J. Sci. Comput., 21 (1999), pp. 666-690.

[8] C.-Y. KAO, S. OSHER, AND J. QIAN, Lax-Friedrichs sweeping scheme for static Hamilton-Jacobi equations, J. Comput. Phys., 196 (2004), pp. 367-391.

[9] G. Kossioris, C. Makridakis, and P. Souganidis, Finite volume schemes for HamiltonJacobi equations, Numer. Math., 83 (1999), pp. 427-442.

[10] S. Osher And C.-W. ShU, High-order essentially nonoscillatory schemes for Hamilton-Jacobi equations, SIAM J. Numer. Anal., 28 (1991), pp. 907-922.

[11] J. QIAN AND W.W. SYMES, An adaptive finite difference method for traveltimes and amplitudes, Geophysics, 67 (2002), pp. 167-176.

[12] E. Rouy And A. Tourin, A viscosity solutions approach to shape-from-shading, SIAM J. Numer. Anal., 29 (1992), pp. 867-884. 
[13] J.A. Sethian, Fast marching methods, SIAM Rev., 41 (1999), pp. 199-235.

[14] J.A. SETHian AND A. Vladimirsky, Fast methods for the Eikonal and related Hamilton-Jacobi equations on unstructured meshes, Proc. Natl. Acad. Sci. USA, 97 (2000), pp. 5699-5703.

[15] J.A. Sethian And A. Vladimirsky, Ordered upwind methods for static Hamilton-Jacobi equations: Theory and algorithms, SIAM J. Numer. Anal., 41 (2003), pp. 325-363.

[16] Y.H.R. Tsai, L.T. Cheng, S. Osher, And H.K. ZhaO, Fast sweeping algorithms for a class of Hamilton-Jacobi problems, SIAM J. Numer. Anal., 41 (2003), pp. 673-694.

[17] J.N. Tsitsiklis, Efficient algorithms for globally optimal trajectories, IEEE Trans. Automat. Control, 40 (1995), pp. 1528-1538.

[18] J.W.J Williams, Heapsort, Comm. ACM, 7 (1964), pp. 347-348.

[19] H.K. ZнаO, Fast sweeping method for eikonal equations, Math. Comp., 74 (2005), pp. 603-627. 
Copyright of SIAM Journal on Scientific Computing is the property of Society for Industrial and Applied Mathematics and its content may not be copied or emailed to multiple sites or posted to a listserv without the copyright holder's express written permission. However, users may print. download, or email articles for individual use. 